\title{
NEXT: A Neutrinoless Double Beta Decay Experiment with a Gaseous Xenon TPC
}

Thorsten Lux*,F. Granena, F. Nova, J. Rico, Institut de Fisica d'Altes Energies, Barcelona, Spain

E-mail: Thorsten.Lux@ifae.es

D.R. Nygren

LBNL, Berkeley, USA

J.A.S. Barata, F.I.G.M. Borges, C.A.N. Conde, T.H.V.T. Dias, L.M.P. Fernandes, E.D.C. Freitas, J.A.M. Lopes, C.M.B. Monteiro, J.M.F.dos Santos, F.P. Santos, L.M.N. Tavora, J.F.C.A. Veloso

Universidade de Coimbra, Portugal

E. Calvo, I. Gil-Botella, P. Novella, C. Palomares, A. Verdugo

CIEMAT, Madrid, Spain

I. Giomataris, E. Ferrer-Ribas

CEA, IRFU, Saclay, France

J.A. Hernando-Morata, D. Martinez, X. Cid

Universidade de Santiago de Compostela, Spain

M. Ball, S. Carcel, A. Cervera, J. Diaz, A. Gil, J.J. Gomez-Cadenas, J. Martin-Albo, F. Monrabal, J. Munoz-Vidal, L. Serra, M. Sorel, N. Yahlali

Instituto de Fisica Corpuscular (IFIC), CSIC - U. de Valencia, Valencia, Spain

R. Esteve Bosch, C.W. Lerche, J.D. Martinez, F.J. Mora, A. Sebastia, A. Tarazona, J.F. Toledo

Instituto ITACA, U. Politecnica de Valencia, Valencia, Spain

M. Lazaro, J.L. Perez, L. Ripoll

U. Politecnica de Valencia, Spain

J.M. Carmona, S. Cebrian, T. Dafni, J. Galan, H. Gomez, F.J. Iguaz, I.G. Irastorza, G. Luzon, J. Morales, A. Rodriguez, J. Ruz, A. Tomas, J.A. Villar

Instituto de Fisica Nuclear y Altas Energias, U. de Zaragoza, Zaragoza, Spain 
Neutrinoless double beta decay searches have the potential to stablish the Majorana nature of the neutrino. NEXT is a new experiment which aims to build a radio pure $100 \mathrm{~kg}$ high pressure electroluminescent Xenon TPC to search for such rare decays. It combines an expected excellent energy resolution with a unique topological signature to achieve a high sensitivity to a light majorana neutrino. The host laboratory will be the new underground site in Canfranc, Spain. This document describes the basic design concept of the NEXT experiment.

European Physical Society Europhysics Conference on High Energy Physics July 16-22, 2009

Krakow, Poland

${ }^{*}$ Speaker. 


\section{Introduction}

Neutrino oscillation experiments have shown that at least two neutrinos have a mass by measuring the square of the mass difference. Another important question about the neutrinos is if they are Majorana particles. In this case the neutrino would be identical to its anti-particle with the consequence that the total lepton number is not conserved. Oscillation experiments cannot give an answer to this, but neutrinoless double beta (DB) decay experiments address this question. DB decays are decays in which a nucleus performs two beta decays in a single process. Within the Standard Model (SM) this process has in the final state two electrons and two anti-neutrinos with the typical spectrum of a multi-body decay. In the case that the neutrino is its own anti-particle, it is possible that the final state contains only two electrons. Since these are carrying all energy released in the process this corresponds under ideal conditions to a delta peak in the energy spectrum. The SM process has already been observed for several isotopes. There is an experiment [1] that has presented a controversial experimental observation of the neutrinoless DB mode. To confirm or unambiguosly exclude this evidence new experiments are needed, which combine a large active mass of DB isotope with excellent control of the backgrounds. This, in turn, requires a very good energy resolution together with any available extra handle over background events.

\section{The NEXT experiment}

NEXT stands for Neutrino Experiment with a Xenon TPC. This experiment is planning to use ${ }^{136} \mathrm{Xe}$ as isotope for a $100 \mathrm{~kg}$ high pressure Time Projection Chamber (HP TPC) ( $\approx 10$ bar) to be operated in the new Spanish underground facility in Canfranc [2]. The use of Xe is for various reasons advantageously: The interesting isotope ${ }^{136} \mathrm{Xe}$ has a relatively large abundance in natural $\mathrm{Xe}(8.9 \%)$ and can be easily enriched by centrifugation at a reasonable cost. In addition it has a high Q value (2480 MeV), the maximal energy the electrons can carry away, allowing a better separation between the DB with neutrinos and the neutrinoless DB in the spectrum. A high Q value also reduces the amount of background coming from radioactive materials. Xe also does not have any other long-living radioactive isotopes which could introduce background. Furthermore it is a noble gas and can be used at the same time for the detection of the charged particles released in the decay. An interesting property of pure Xe is that it is not only ionized easily when a charged particle traverses but that it emits during that process also large amounts of primary scintillation light $(175 \mathrm{~nm})$ which can be used for determining the start-of-event time.

\section{The detector concept}

The detector concept is motivated by the requirement of having an excellent energy resolution $(\leq 1 \%$ at $2.48 \mathrm{MeV}$ ) and a handling for the background suppression. The former is the only possibility to distinguish between the intrinsic background coming from $\beta \beta^{2 v}$ and $\beta \beta^{0 v}$ events and also helps to separate external background from the signal, while the latter reduces the contribution from external background. The proposed design is based on ideas developed by D. Nygren [3] and D. Sinclair [4] and is called the SOFT (separated-optimized functions for tracking) approach. The keypoint is to have a specific readout for both applications, tracking and energy measurement. 

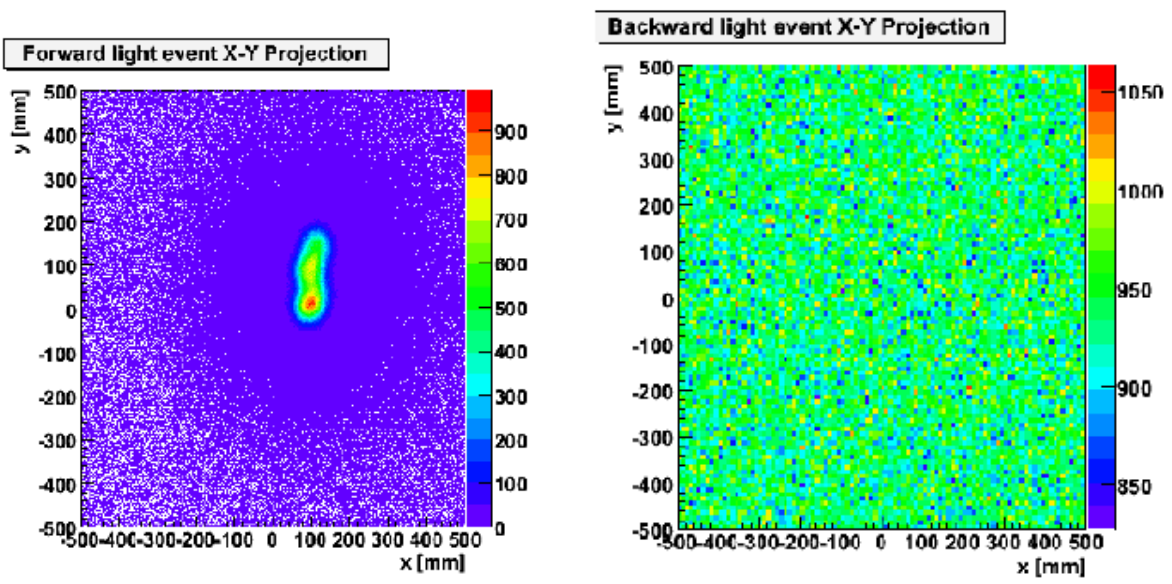

Figure 1: On the left a simulated signal event as it is seen by the tracking readout. In contrary to this the light loses under ideal conditions all information about its origin when measured behind the cathode.

It was shown that the best energy resolution in a Xe detector can be achieved with a electroluminisence (EL) readout [5]. In such a detector the primary electrons released in the ionization are drifting in an external electric field to two parallel meshes separeted by a few mm. A potential difference between these two meshes is choosen such that the energy of the electrons are kept below the ionization potential of the Xe but high enough to excited the Xe. The excited Xe afterwards decays emitting isotropically secondary scintillation light of $175 \mathrm{~nm}$ which can be detected with photosensors. The advantage is that this is, in contrast to a charge readout, a linear process yielding smaller fluctuations and therefore better energy resolutions. Energy resolutions of $7.9 \%$ for 5.9 $\mathrm{keV}$ and gains of $\approx 1000$ were achieved with EL in Xe [6].

The idea is now to use the EL photons emitted towards the hemisphere of the anode for the tracking, while a set of PMTs mounted behind the transparent cathode would measure the photons emitted in the opposite direction. An additional advantage of this idea under ideal conditions (rectangular shape of the TPC, walls with fully specular reflectivity) is that the light is smeared homogenously over the area (see fig. 1 allowing to reduce the required number of PMTs drastically leading to lower costs and less external background. To what extent this feature is preserved under realistic conditions is currently topic of a study. The PMTs serve at the same time for creating the trigger signal from the primary scintillation light. For the design choices also important is the scalability of the detector to the ton scale.

\section{Tracking}

A significant amount of external background can be ejected by tracking. While the signature of a DB decay is a track with two blobs at the ends the pattern e.g. of a photon entering the chamber and producing there a Compton electron consists of a track with only one blob. For the tracking three readout options are investigated: One forsees the use of Micromegas (MM). As long as it turns out that the energy resolution directly achieved with MM is not sufficient, the MM would be placed 
in this scheme behind the parallel meshes. The electrons first produce the secondary scintillation light and are then guided to the MM where a charge amplification takes place. The advantages of MM are that they can be produced cost-effective in modules of $\approx 35 \times 35 \mathrm{~cm}$ [7] and the use of standard readout electronics. The stable operation of MM in pure Xe at high pressures as also the effect of scintillation light produced during the charge amplification on the energy resolution measurement by the PMTs are under investigation. Another option for the tracking is the use of Multi-Pixel Photon Counters (MPPCs). These relatively new devices are suitable to count single photons while the costs for a single sensor is very low. In addition due to the high internal gain the use of simpler electronics might be possible. The MPPCs are $\approx 1 \times 1 \mathrm{~mm}^{2}$ ) large. It is under study if it is sufficient to cover only $1 \%$ of the area by placing them with a pitch of $1 \mathrm{~cm}$ or if the MPPCs could be coupled to Winston Cones. Also the effect of their limited dynamic range, their noise rate and their large temperature dependency on the pattern recognition capability are investigated. Another option for the tracking are avalanche photodiodes (APDs). These are tested already for some years successfully in small test chambers [8]. The sensors are slightly modified commercial products which can be provided in large quantities by industry. The costs per sensor are the main drawback of this technology at the moment.

\section{Summary and Outlook}

NEXT is a new $\beta \beta^{0 v}$ experiment planned to be operated at the Canfranc Underground Laboratory. The collaboration aims to build a $100 \mathrm{~kg}$ HP TPC (NEXT-100) filled with enriched ${ }^{136}{ }^{\mathrm{Xe}}$. The detector will be radio-pure to fullfil the stringent requierments on the background suppression. The approach for the detector design is based on the separation of tracking and energy funtions inside the TPC. Simulations indicate that this approach will provide the excellent energy resolution and the background rejection capability which are required by the physics program. It is expected that NEXT-100 can cover the effective mass region down to $100 \mathrm{meV}$ after 5 years of operation.

\section{Acknowledgements}

We acknowledge the support received by the CONSOLIDER INGENIO Project CSD20080037 (CUP)

\section{References}

[1] H. V. Klapdor-Kleingrothaus, et al. Mod. Phys. Lett. A 16 (2001) 2409

[2] F. Granena et al.,LoI, arXiv:0907.4054 [hep-ex].

[3] D. Nygren, Nucl. Instrum. Meth. A 581 (2007) 632.

[4] D. Sinclair, Talk given on the 4th Symposium on large TPCs for low energy rare event detection, 2009

[5] E. Aprile et al., Noble Gas Detectors, Wiley-VCH Verlag GmbH \& Co. KGaA, 2006

[6] C. M. B. Monteiro et al., JINST 2 (2007) P05001

[7] M. Di Marco [T2K Collaboration], J. Phys. Conf. Ser. 65 (2007) 012019.

[8] L. M. P. Fernandes et al., JINST 2 (2007) P08005 